\section{PL.41 SPECIFIC MICRORNAS ARE DIFFERENTIALLY EXPRESSED IN LABOURING AND NON-LABOURING HUMAN MYOMETRIUM AT TERM}

doi:10.1136/archdischild-2013-303966.224

JR Cook, DA MacIntyre, P Bennett, V Terzidou. Parturition Research Group, Imperial College, London, UK

Introduction MicroRNAs (miRNAs) are small non-coding singlestranded RNA molecules which down-regulate gene expression at the post-transcriptional level. Their influence is thought to be vast and it is estimated that approximately $30 \%$ of the human genome may be regulated by miRNAs. Recent studies in fetal membranes suggest differential expression of miRNAs with advancing gestation or histological evidence of chorioamnionitis (1), which is one of the primary causes of preterm labour. Further, cyclo-oxygenase (COX-2), which synthesises prostaglandin which in turn modulates uterine contractions has been shown to be regulated at the posttranscriptional level through changes in specific microRNAs (2)

Methods and Results Myometrial biopsies were collected from women undergoing caesarean section before and after the clinical onset of labour ( $\mathrm{n}=9$, each group). Total RNA was extracted using RNA-STAT 60. Samples were further characterised by assessing COX-2 expression using qRT-PCR with GAPDH as an endogenous control; a 'non-labouring' sample with high COX-2 expression was excluded. RNA samples were labelled using the miRCURYTM Hy3/ Hy5 power labelling kit and hybridised on the miRCURYTM LNA array. The results of the array were validated with qRT PCR. Principle component analysis of co-variance between samples demonstrated variance contributing to phenotype at the third component level. Orthagonal partial least squares discriminatory analysis identified eight microRNAs whose variance is related to the onset of labour. Six had reduced expression with labour and two were increased.

Conclusion MiRNAs may have a potential role regulating gene expression at the onset of labour.

\section{REFERENCES}

1. Montenegro D et al. J Pathol 2009;217(1):113-21.

2. Harper KA et al. Complexity of COX-2 gene regulation. Biochem Soc Trans 2008; 36(Pt 3):543-5.

\section{PL.42 IN UTERO TRANSFER: 'IT'S SOMETHING YOU HAVE TO PUT UP WITH': A QUALITATIVE EXPLORATION OF ITS IMPACT ON FAMILIES}

doi:10.1136/archdischild-2013-303966.225

${ }^{1} \mathrm{~L}$ Porcellato, ${ }^{2} \mathrm{G}$ Masson, ${ }^{2} \mathrm{~F} 0$ 'Mahony, ${ }^{2} \mathrm{E}$ Perkins, ${ }^{3} \mathrm{~S}$ Jenkinson, ${ }^{3} \mathrm{~T}$ Vanner, ${ }^{3} \mathrm{~K}$ Cheshire 'Liverpool John Moores University, Liverpool, UK; ' ${ }^{2}$ niversity Hospital of North Staffordshire NHS Trust, Newcastle Under Lyme, UK; ${ }^{3}$ The Royal Wolverhamtpon Hospitals NHS Trust, Wolverhampton, UK

In utero transfer (IUT) is a necessary facet of contemporary obstetrics, enabling the appropriate level of care for neonates (BAPM 2001). However, quantitative research has demonstrated that IUT can cause stress and anxiety and potentially impedes future pregnancies (Walker 2000). Although the impact of IUT on the families of expectant mothers has been assessed by Jackson et al, (2010) in a Scottish-wide audit, the issue has been largely ignored in England. It is important to examine IUTs from the service users' perspective, to ensure services are responsive to need and engender a positive birth experience.

A small scale qualitative study was undertaken, to gain insight into the IUT process as experienced by women and their families, with a view to informing maternal care policy and practise. Semi structured interviews were carried out with 8 family members and 15 women who had been transferred into 2 tertiary obstetric centres in England between Aug 2010 and Dec 2011. Hour long digitally recorded interviews were conducted and thematically analysed using Nvivo 9. Results highlighted that the impact of IUT on families was primarily psychological, physical, social and financial; fathers were perceived to be the most affected by the process. There was resigned acceptance of the need for IUT but issues around lack of information about the transfer hospital, inflexible visiting hours and the inconvenience to visitors had a negative impact on families and need to be addressed, to improve the IUT experience for future service users.

\section{REFERENCES}

BAPM (Dec 2001) Standards for Hospitals Providing Neonatal and High Dependency Care (2 Edition).

Jackson L, McLean D, Wilson AM, Skeoch C, An evaluation of the financial and emotional impact of in utero transfers upon families: a Scotland-wide audit. Infant, 6:2 (March 2010).

Walker J (2000) Women's Experiences of Transfer from a Midwife-led to a Consultantled Maternity unit in the UK During Late Pregnancy and Labour. JMWH 45: 2, 161-168.

\section{PL.43 CERVICAL COMPLIANCE AND SMOOTH MUSCLE}

doi:10.1136/archdischild-2013-303966.226

C Mowbray, SC Robson, JN Bulmer, MC Smith. Institute of Cellular Medicine, Newcastle University, Newcastle Upon Tyne, UK

Background Cervical weakness is associated with late miscarriage and extreme preterm birth. Early studies reported higher cervical smooth muscle content in women who experienced second trimester loss. Contemporary studies have suggested structural composition is not related to pre-labour cervical remodelling. The aim of the present study was to explore the relationship between human cervical structure and biomechanical characteristics.

Methods Cervical biopsies were taken from 17 non-pregnant women at the time of hysterectomy. Samples were bisected longitudinally and one portion mounted on a myograph (Danish Systems). The stretch-strain characteristics were recorded using Windaq software. The non-stretched portion was fixed and immunohistologically stained with orcein (elastin) and immunostained for smooth muscle actin [SMA]. Smooth muscle and elastin content were then assessed using Photoshop image analysis. 3 biopsies were taken from each of 4 hysterectomy specimens to assess intra-subject variability. Results The proportion of both smooth muscle and elastin content of cervical biopsies showed a wide variation $(5-47 \%$ and $9-47 \%$, SMA and elastin, respectively). Furthermore, there was considerable intra-subject variability with as much as a 2.4 fold difference in SMA content. Both SMA and elastin content correlated with biopsy compliance $(\mathrm{R}=0.3, \mathrm{p}<0.01$, and $\mathrm{R}=0.4, \mathrm{p}<0.01$ respectively).

Conclusion The biomechanical properties of human cervical biopsies are influenced by the smooth muscle and elastin content of the specimen. Histological and biomechanical characteristics of human cervical biopsies cannot be assumed to be reliably representative of the whole specimen. These findings may explain why previous studies have failed to find a relationship between biopsy characteristics and clinical history.

\section{PL.44 REPEAT USE OF DINOPROSTONE PESSARY AFTER FAILED INDUCTION OF LABOUR}

doi:10.1136/archdischild-2013-303966.227

'M Umoren, ${ }^{2}$ S Relph, ${ }^{2} \mathrm{~A}$ Fakokunde. 'St George's Medical School, Grenada, Grenada; ${ }^{2}$ North Middlesex University Hospital, London, UK

Introduction There is no guideline advising on the management of women in whom induction of labour with first dinoprostone pessary has failed. Repeat cervical ripening with a second pessary is a commonly used management option. 